Article

\title{
Comparative Study of Validity and Reliability of Two Handgrip Dynamometers: K-Force Grip and Jamar
}

\author{
Thomas Nikodelis ${ }^{1, * \mathbb{D}}$, Stratos Savvoulidis ${ }^{1}$, Petros Athanasakis ${ }^{1}$, Christos Chalitsios ${ }^{1}$ (D) and \\ Theodoros Loizidis ${ }^{2}$ \\ 1 Biomechanics Lab, Department of Physical Education and Sport Science, Aristotle University of Thessaloniki, \\ 54124 Thessaloniki, Greece; stratoss@phed.auth.gr (S.S.); petratha@phed.auth.gr (P.A.); \\ cchalits@phed.auth.gr (C.C.) \\ 2 Department of Physical and Rehabilitation Medicine, Saint Loukes Hospital, 55236 Thessaloniki, Greece; \\ loizidis@yahoo.com \\ * Correspondence: nikmak@phed.auth.gr; Tel.: +30-2310992202
}

check for updates

Citation: Nikodelis, T.; Savvoulidis, S.; Athanasakis, P.; Chalitsios, C.; Loizidis, T. Comparative Study of Validity and Reliability of Two Handgrip Dynamometers: K-Force Grip and Jamar. Biomechanics 2021, 1, 73-82. https://doi.org/10.3390/ biomechanics1010006

Received: 20 January 2021

Accepted: 23 March 2021

Published: 25 March 2021

Publisher's Note: MDPI stays neutral with regard to jurisdictional claims in published maps and institutional affiliations.

Copyright: (C) 2021 by the authors Licensee MDPI, Basel, Switzerland. This article is an open access article distributed under the terms and conditions of the Creative Commons Attribution (CC BY) license (https:// creativecommons.org/licenses/by/ $4.0 /)$.

\begin{abstract}
Background: Handheld dynamometers are used for diagnosis and rehabilitation at several pathologies. Grip force is essential for a person's quality of life. Most dynamometers are not equipped with rehabilitation or training protocols, although the bibliography highlights this need. Objective: To compare the validity and reliability of Jamar and K-Force Grip dynamometers in patients with shoulder impingement syndrome and healthy people. Methods: Concurrent validity was assessed using known weights. Forty-four (44) patients and 69 healthy adults performed three maximum repetitions in random order with each hand, at each instrument. Reliability was evaluated using ICCs for each instrument separately, and between the two instruments. The differences between the two dynamometers were evaluated using repeated $t$-tests. Results: Concurrent validity of the two instruments did not reveal fix or proportional bias. Analyses for reliability yield high correlation coefficients for both groups with the lower one being between the two instruments $r=0.90, p<0.05$, $(0.72-0.91)$ for the healthy group. $t$-tests showed that all participants had lower force values when using the K-Force Grip $(p<0.05)$. Conclusion: Both dynamometers were reliable in measuring hand grip strength for both groups. K-Force Grip was reliable and valid with respect to Jamar that was used as a benchmark. The different handle dimensions between the two dynamometers may be the reason for the different force values.
\end{abstract}

Keywords: hand grip force; dynamometers; rehabilitation protocols; shoulder impingement

\section{Introduction}

Handgrip dynamometers are used for a plethora of different objectives. The most popular of them are assessing overall strength to compare muscle function across populations and evaluate progression of wasting conditions, like sarcopenia, or even identify potential deficits [1,2]. Handgrip dynamometers are also used as functional assessment tools to measure the strength of different muscles, directly related with a pathological condition, like rotator cuff strength [3]. Regarding the rehabilitation process, handgrip dynamometers are mainly used for evaluation purposes, such as tracking overall progress through rehabilitation [4] or testing the effect of a specific rehabilitation protocol targeting a problem related to grip strength like shoulder impingement syndrome [5]. Adequate handgrip strength is necessary for independent and quality daily living [6]. Yet, coordinated handgrip behavior and grip strength can be lost in different pathological conditions. This is supported by the results of a previous study [7] showing that multi-morbidity is statistically negatively related to grip strength.

Most of the handgrip dynamometers are not equipped with an integrated module in order to be used in rehabilitation protocols, such as scaling force targets, proprioception enhancement tasks, or even Visio-motor coordination tasks despite the fact that there is a 
growing body of evidence concerning the necessity of such interventions in the course of rehabilitation [8]. In many studies, the use of new technological equipment has been found to be the most preferable asset in rehabilitation $[9,10]$.

K-Force Grip ${ }^{\circledR}$ Kinvent 2016 is a dynamometer used in the evaluation and rehabilitation of handgrip strength that provides real time biofeedback on a Tablet or a Smartphone, allowing the therapist to set strength objectives and motivate the patient through the process of rehabilitation. It is efficient and very fast at getting from the results. Testing and reporting the export is concluded in $15 \mathrm{~s}$ as there is no need to transcript values to spreadsheets and print them. It also provides the choice of interactive games based on variations of force levels through the brand's games suite. The therapist can follow up the patient's progress through K-Force Grip's interactive database. K-Force Grip enables the therapist to create training protocols for the patient based on biofeedback sensory information taking full advantage of the whole force time series and not only a distinct value. Although this tool is already being used by the therapists and can be very promising in rehabilitation, it has not yet been tested for reliability and agreement with other devices used in the literature.

There are several studies that have tried to validate various grip strength devices. Most of them compared the instrument to be validated with the Jamar dynamometer [11-13]. More specifically, Hogrel et al. [14] measured 346 healthy subjects with the MyoGrip and the Jamar dynamometers and concluded that Myogrip is reliable for use. Other researchers investigated the agreement of the instruments in pathological groups $[15,16]$. Jamar is used in this study as the gold standard.

Most of the studies focused on ICC (intra-class correlation coefficients) $[17,18]$ with 95\% confidence intervals to measure intra-instrument and inter-instrument reliability, while concurrent validity is assessed with known weights. Thus, correlation coefficients are obtained [18]. In the cases that differences were found in the measurement values between the instruments, the practical significance of those differences was acquired [18].

The purpose of the present study was to compare Jamar and K-Force Grip for concurrent validity as well as intra-instrument and inter-instrument reliability and difference in measured values for both patients suffering from shoulder impingement syndrome and healthy adults.

\section{Materials and Methods}

A total of 113 individuals voluntarily participated in the study. Forty-four (44) individuals suffering from shoulder impingement syndrome (pain group), 21 males, 23 females, and sixty-nine healthy individuals, students of physical education (healthy group), 30 males, and 39 females participated in the study. Their anthropometric characteristics are reported in Table 1.

Table 1. Anthropometric characteristics of the participants.

\begin{tabular}{ccccc}
\hline & Age & Body Weight & Height & Body Mass Index (BMI) \\
\hline Pain group (male) & $51.6 \pm 13$ years & $79.5 \pm 12.1 \mathrm{~kg}$ & $174.2 \pm 8 \mathrm{~cm}$ & $27.1 \pm 7 \mathrm{~kg} / \mathrm{cm}^{2}$ \\
Pain group (female) & $58 \pm 15.7$ years & $63.2 \pm 11.5 \mathrm{~kg}$ & $159.2 \pm 11 \mathrm{~cm}$ & $25.2 \pm 6.3 \mathrm{~kg} / \mathrm{cm}^{2}$ \\
Healthy group (male) & $19.9 \pm 1.6$ years & $74.52 \pm 9.1 \mathrm{~kg}$ & $177.8 \pm 6 \mathrm{~cm}$ & $23.6 \pm 2.2 \mathrm{~kg} / \mathrm{cm}^{2}$ \\
Healthy group (female) & $19.4 \pm 0.5$ years & $56.62 \pm 6.6 \mathrm{~kg}$ & $163.8 \pm 5 \mathrm{~cm}$ & $22 \pm 2.3 \mathrm{~kg} / \mathrm{cm}^{2}$ \\
\hline
\end{tabular}

Participants of the pain group were volunteers recruited during their visit to a medical center. Participation had to meet the following criteria: (i) Presence of shoulder pain and painful arc for 15 days up to three months (subacute stage), as longer duration is expected to have degenerative changes [19], (ii) impingement syndrome after physical examination at the site of treatment with musculoskeletal ultrasound (Sonoline G20), (iii) diagnosis of impingement syndrome and not joint arthritis (AC, Shoulder), (iv) clinical signs of nerve pressure or any red flag of other pathology, (v) no sign of neurological deficit, spine trauma, or surgery to the cervical or shoulder area, (vi) absence of rheumatic or infectious diseases, psychiatric or cancer history, metabolic diseases (diabetes or thyroid), coagulopathies or use of anticoagulants, and neuromuscular degenerative diseases, and (vii) presence of 
painful arc on the movement of the shoulder and to be positive for an anterior slide test, apprehension test, and Hawkin's test or impingement sign [20]. Patients had a different clinical picture, but they all had to have pain on the suprascapular tendon and a positive sign on the supraspinatus tendon. Patients with frozen shoulders were excluded from the study. Those who had received anti-inflammatory drugs for the last 5 days, underwent surgery or arthroscopy in the shoulder in question, or a corticosteroid injection in the joint during the last month before the study were also excluded.

From a larger pool that visited the medical centre in a period of 2 months, 44 individuals had the above characteristics and agreed to participate. Those physical education students who responded to the call, agreed to participate and were included in the study. All participants were informed in advance about the purpose of the study and they were asked to sign a consent form. The study was approved by the ethics committee of the University (Approval number EC-13/2020).

The two instruments have a similar measuring capacity $(90 \mathrm{~kg})$. Yet, they have differences regarding their weight, with Jamar being more than 3 times heavier (490 gr vs. $150 \mathrm{gr}$ ) and their dimensions (Jamar: $60 \times 140 \times 240 \mathrm{~mm}$, K-Force Grip: $40 \times 45 \times$ $120 \mathrm{~mm}$ ). Jamar also has a replaceable battery while K-Force Grip has a rechargeable one. An important difference is the handle position. Jamar has an adjustable handle position while K-Force Grip has a fixed one.

\subsection{Concurrent Validity}

In order to check the instruments' concurrent validity, free weights of known mass (commercial plates used in weightlifting) that were also weighted prior to the test, were used. Both dynamometers secured within a working bench and a series of combinations among the known weights, ranging from 5 to $60 \mathrm{~kg}$, hanged from the middle of the handles, using a hook attached to a loop of rope. In total, 20 different weights were hanged. For each weight, the equivalent measure from the dynamometric device was stored.

\subsection{Strength Assessment}

The participants' maximum isometric hand grip strength was measured using K-Force Grip $^{\circledR}$ (Kinvent, Monpellier, France) \& Jamar ${ }^{\circledR}$ Plus + Digital Hand Dynamometer (Paterson Medical ${ }^{\circledR}$, Green Bay, WI, USA) (Figure 1a,b).

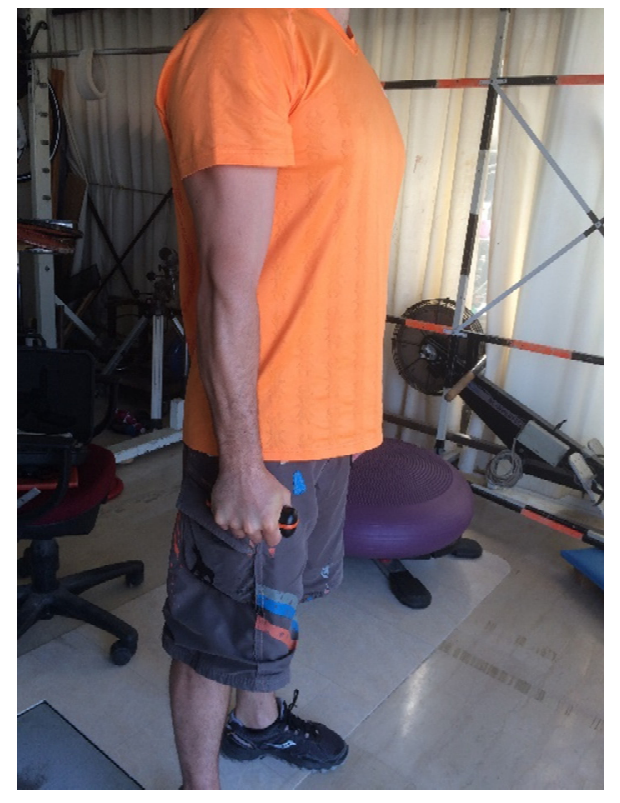

(a)

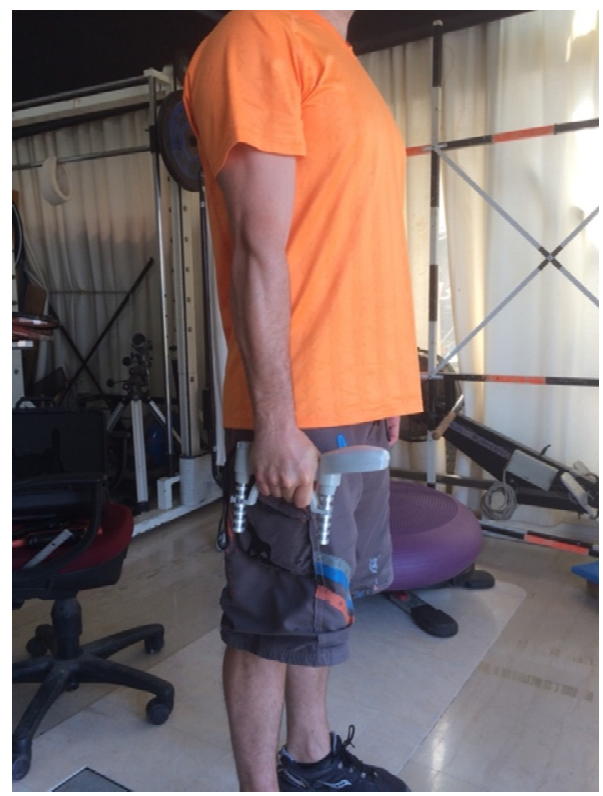

(b)

Figure 1. (a) Standing position with K-Force Grip Dynamometer. (b) Standing position with Jamar Dynamometer. 
After a short familiarization process with the dynamometers, all the participants performed three trials with each hand, using both instruments. They maintained a standing position with the elbow joint in extension (Figure 1a,b). The K-Force Grip device has an ergonomic elliptic shape for a better grabbing with an external diameter from 37 to $45 \mathrm{~mm}$. Taking into consideration these dimensions, the second position of the Jamar handle was used since it was closer to the diameter of the K-Force Grip. The width along the handle of Jamar was measured to be from $44 \mathrm{~mm}$ to $49 \mathrm{~mm}$. The tests were held at random order. Each trial test lasted 3 seconds while there was at least a one-minute interval between trials. All trials were held on the same day during morning hours for each participant. All measurements for the healthy group were performed in the same week while, for the pain group, all measurements were performed in a period of one month. The standing testing position with a full elbow extension was selected as it has shown excellent test-retest reliability in the literature [21,22]. Through consecutive trials with each hand separately for each dynamometer, the instrument reliability (intra-instrument) for the specific groups at the selected position, was checked. There was no verbal encouragement, apart from the instruction of "start" and "stop". The time was kept with the use of an electronic chronometer for Jamar and the official software regarding K-Force Grip. All measurements were supervised by the same person.

\subsection{Statistical Analysis}

The data were originally checked for normality in their distribution using the KolmogorovSmirnov (K-S) test.

To investigate if fixed and proportional bias were present at a concurrent validity test within repeated measures of each instrument and between the two instruments, the method of ordinary least products regression (OLP) was used [23].

Intra-instrument reliability was tested using an ICC (two-way random with absolute agreement) for both instruments using the three trials for each hand. Inter-instrument reliability was also tested using the same ICC test correlating the mean values from each hand between the two dynamometers. At all cases, single measure ICC values were used. Paired $t$-tests were also held between the two dynamometers for the mean values of each hand, in order to check for differences in the measurements. According to the scores of the participants and their number in each group, the Cohen's D effect size was calculated for every comparison. The level of significance for all analyses was set at $p<0.05$. All analyses were held using a SPSS 21 statistical package. All procedures were repeated twice, which involves once for each group of participants.

\section{Results}

All the variables had approximately normal distribution since they had a $p$ value $>0.05$ in the K-S test. The OLP test confirmed that there was no bias in the data except the case of the mean values for the healthy group of a proportional bias was found (Table 2).

Exploratory analysis revealed that there were five outliers. At least two cases of participants had extreme values with more than $20 \mathrm{~kg}$ of difference for Jamar among the three trials (i.e., Jamar 20, 42, and $38.3 \mathrm{~kg}$ ). There were also similar extreme differences in $\mathrm{K}$-grip around $10 \mathrm{~kg}$. For comparative reasons, the last row of Table 2 shows the results when the outliers were removed from the analysis. The proportional bias disappeared. Yet a significant fixed bias was found.

A scatterplot concerning concurrent validity to show the data dispersion around the line of best fit is shown in Figure 2 for each instrument. 
Table 2. Ordinary least products regression (OLP) test. Fixed bias is present if the confidence interval does not include 0 and proportional if it does not include 1 . The existence of bias is marked with an asterisk.

\begin{tabular}{|c|c|c|c|c|}
\hline \multicolumn{5}{|c|}{ Concurrent Validity (Against Fixed Mass) } \\
\hline \multirow[t]{2}{*}{ Variable } & \multicolumn{2}{|c|}{ Fixed Bias } & \multicolumn{2}{|c|}{ Proportional Bias } \\
\hline & Intercept & $95 \% \mathrm{CI}$ & Slope & $95 \%$ CI \\
\hline Grip & 0.004 & $-0.142-0.151$ & 0.998 & $0.993-1.003$ \\
\hline Jamar & -0.048 & $-0.168-0.071$ & 0.999 & $0.995-1.004$ \\
\hline \multicolumn{5}{|c|}{ Within instruments reliability } \\
\hline \multirow[t]{2}{*}{ Variable } & \multicolumn{2}{|c|}{ Fixed bias } & \multicolumn{2}{|c|}{ Proportional bias } \\
\hline & Intercept & $95 \% \mathrm{CI}$ & Slope & $95 \% \mathrm{CI}$ \\
\hline Grip Left 1-2 & -1.04 & $-4.42-2.03$ & 1.06 & $0.96-1.16$ \\
\hline Grip Left 2-3 & 0.6 & $-2.81-3.68$ & 1.01 & $0.91-1.12$ \\
\hline Grip Left 1-3 & -0.53 & $-6.65-4.61$ & 1.07 & $0.91-1.26$ \\
\hline Grip Right $1-2$ & -2.62 & $-5.73-0.23$ & 1.07 & $0.99-1.17$ \\
\hline Grip Right $2-3$ & 0.79 & $-2.45-3.75$ & 0.96 & $0.87-1.06$ \\
\hline Grip Right $1-3$ & 1.8 & $-5.87-1.84$ & 1.03 & $0.93-1.15$ \\
\hline Jamar Left 1-2 & -1.54 & $-9.43-4.99$ & 1.03 & $0.85-1.23$ \\
\hline Jamar Left 2-3 & 1.51 & $-1.61-4.4$ & 0.96 & $0.88-1.04$ \\
\hline Jamar Left 1-3 & 0.12 & $-5.94-5.34$ & 0.98 & $0.84-1.14$ \\
\hline Jamar Right 1-2 & 2.67 & $-1.15-6.17$ & 0.93 & $0.84-1.02$ \\
\hline Jamar Right 2-3 & 1.57 & $-2.06-4.92$ & 0.95 & $0.87-1.05$ \\
\hline Jamar Right 1-3 & 4.21 & $-0.27-8.24$ & 0.91 & $0.82-1.01$ \\
\hline \multicolumn{5}{|c|}{ Between instruments reliability } \\
\hline \multirow[t]{2}{*}{ Variable } & \multicolumn{2}{|c|}{ Fixed bias } & \multicolumn{2}{|c|}{ Proportional bias } \\
\hline & Intercept & $95 \% \mathrm{CI}$ & Slope & $95 \%$ CI \\
\hline $\begin{array}{l}\text { Pain group (mean } \\
\text { values Jamar-Grip) }\end{array}$ & 0.35 & $-3.77-4.00$ & 0.93 & $0.84-1.02$ \\
\hline $\begin{array}{l}\text { Healthy group (mean } \\
\text { values Jamar-Grip) }\end{array}$ & 1.52 & $-2.33-4.97$ & 0.79 & $0.68-0.91$ * \\
\hline $\begin{array}{l}\text { Healthy group (mean } \\
\text { values Jamar-Grip) } \\
\text { outliers removed }\end{array}$ & -5.13 & $(-8.45)-(-2.07) *$ & 0.89 & $0.83-1.01$ \\
\hline
\end{tabular}

Intra-instrument and inter-instrument reliability showed large values of ICC for both hands at both instruments for both groups. The detailed results for each group are shown in Table 3.

Table 3. Intra-class correlation coefficients (ICC) with lower and upper bounds of a $95 \%$ confidence interval for the pain group.

\begin{tabular}{|c|c|c|c|c|c|c|c|c|}
\hline $\begin{array}{c}\text { ICC } \\
95 \% \text { CI }\end{array}$ & \multicolumn{4}{|c|}{ Pain Group } & \multicolumn{4}{|c|}{ Healthy Group } \\
\hline \multirow{2}{*}{$\begin{array}{l}\text { Intra-instrument } \\
\text { reliability }\end{array}$} & $\begin{array}{l}\text { Jamar } \\
\text { affected }\end{array}$ & $\begin{array}{l}\text { Jamar } \\
\text { healthy }\end{array}$ & $\begin{array}{l}\text { K-Force } \\
\text { Grip } \\
\text { (affected) }\end{array}$ & $\begin{array}{c}\text { K-Force } \\
\text { Grip } \\
\text { (healthy) }\end{array}$ & Jamar left & Jamar right & $\begin{array}{c}\text { K-Force } \\
\text { Grip } \\
\text { (left) }\end{array}$ & $\begin{array}{l}\text { K-Force } \\
\text { Grip (right) }\end{array}$ \\
\hline & $\begin{array}{c}0.977 \\
(0.956- \\
0.989)\end{array}$ & $\begin{array}{c}0.983 \\
(0.962- \\
0.993)\end{array}$ & $\begin{array}{c}0.972 \\
(0.946- \\
0.987)\end{array}$ & $\begin{array}{c}0.96 \\
(0.923- \\
0.981)\end{array}$ & $\begin{array}{c}0.929 \\
(0.895- \\
0.954)\end{array}$ & $\begin{array}{c}0.946 \\
(0.919- \\
0.965)\end{array}$ & $\begin{array}{c}0.941 \\
(0.911- \\
0.963)\end{array}$ & $\begin{array}{c}0.949 \\
(0.923- \\
0.967)\end{array}$ \\
\hline \multirow{2}{*}{$\begin{array}{l}\text { Inter-instrument } \\
\text { reliability }\end{array}$} & \multicolumn{2}{|c|}{$\begin{array}{c}\text { Jamar-K-Force Grip } \\
\text { affected }\end{array}$} & \multicolumn{2}{|c|}{$\begin{array}{c}\text { Jamar-K-Force Grip } \\
\text { (healthy) }\end{array}$} & \multicolumn{2}{|c|}{$\begin{array}{c}\text { Jamar- } \\
\text { (left) }\end{array}$} & \multicolumn{2}{|c|}{$\begin{array}{c}\text { Jamar-K-Force Grip } \\
\text { (right) }\end{array}$} \\
\hline & \multicolumn{2}{|c|}{$0.979(0.911-0.993)$} & \multicolumn{2}{|c|}{$0.911(0.706-0.959)$} & \multicolumn{2}{|c|}{$0.91(0.69-0.918)$} & \multicolumn{2}{|c|}{0.907 (0.728-0.9134) } \\
\hline
\end{tabular}




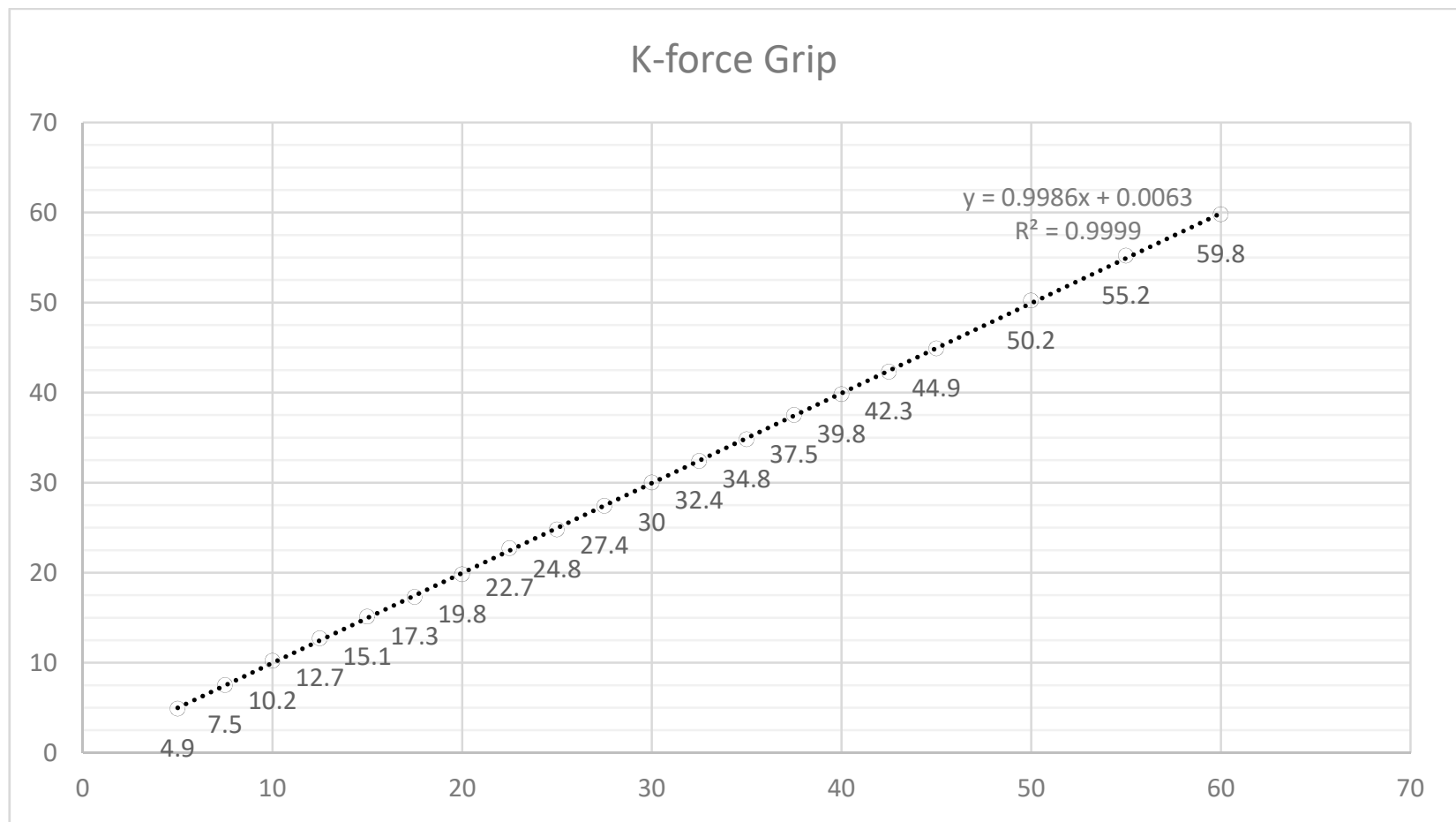

(a)

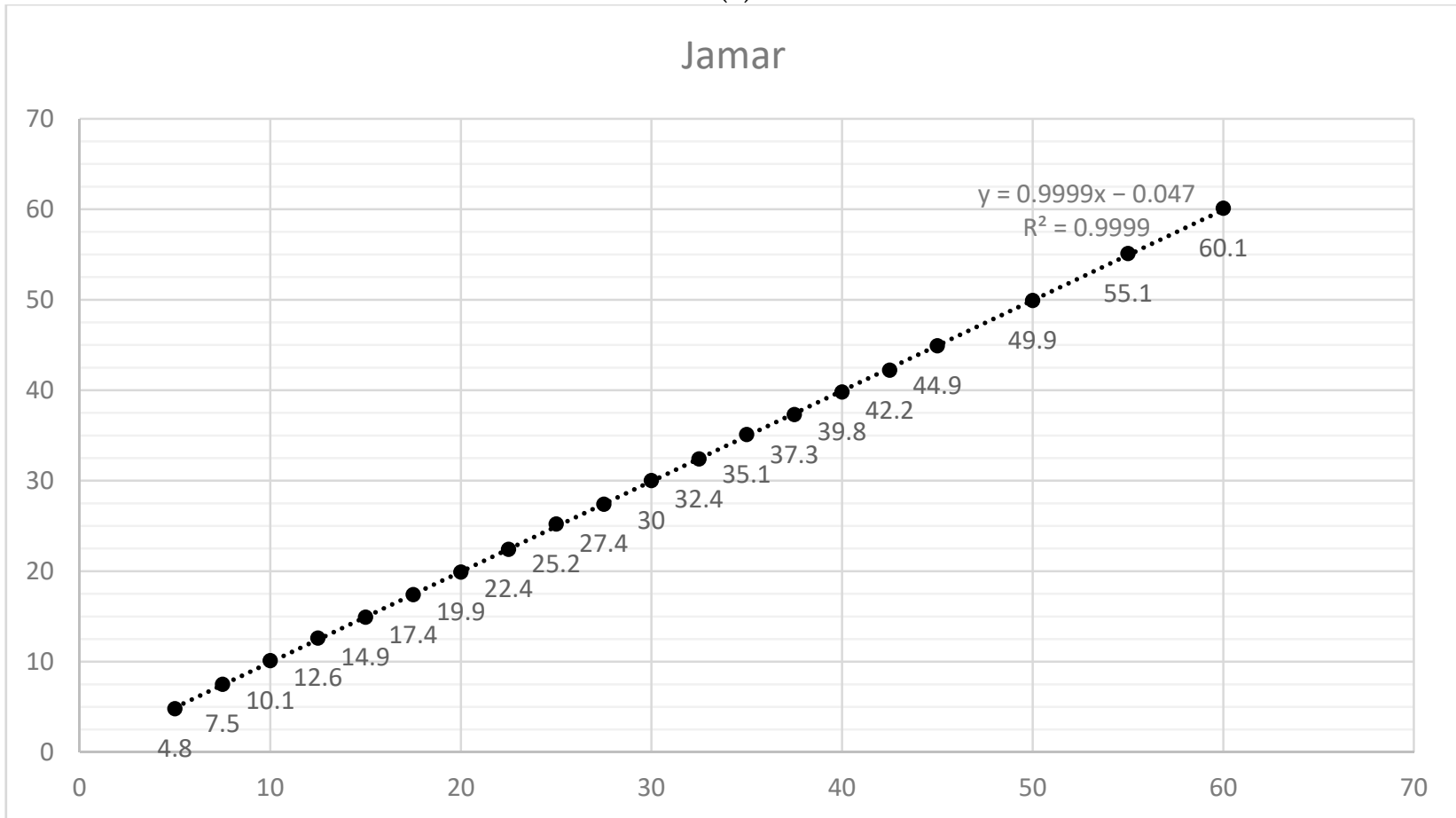

(b)

Figure 2. (a) Data dispersion of 20 measures from known weights for the K-Force Grip Dynamometer (horizontal axis represents known weights, vertical measured values in $\mathrm{kg}$ ). (b) Data dispersion of 20 measures from known weights for Jamar Dynamometer (horizontal axis represents known weights and vertical measured values in $\mathrm{kg}$ ).

Paired $t$-tests between the two dynamometers for the mean values of each hand, showed that the two devices differed significantly for both the affected arm $(p<0.05)$ and the healthy hand $(p<0.05)$ regarding the pain group, and for both the left hand $(p<0.05)$ 
and the right hand $(p<0.05)$ regarding the healthy group, with K-Force Grip having smaller values than Jamar dynamometer at all cases. The calculated effect size was at a medium with the pain group having lower values. The descriptive values and the Cohen's D effect size appear in Table 4.

Table 4. Descriptive values used for the $t$-tests and Cohen's D effect size for every pair of comparisons.

\begin{tabular}{|c|c|c|c|c|c|c|c|}
\hline \multicolumn{4}{|c|}{ Pain Group } & \multicolumn{4}{|c|}{ Healthy Group } \\
\hline Jamar affected & $\begin{array}{l}\text { K-Force Grip } \\
\text { affected }\end{array}$ & Jamar healthy & $\begin{array}{l}\text { K-Force Grip } \\
\text { healthy }\end{array}$ & Jamar left & $\begin{array}{c}\text { K-Force Grip } \\
\text { left }\end{array}$ & Jamar right & $\begin{array}{c}\text { K-Force Grip } \\
\text { right }\end{array}$ \\
\hline $32.1 \pm 17 \mathrm{~kg}$ & $27.3 \pm 13.8 \mathrm{~kg}$ & $32.6 \pm 15.3 \mathrm{~kg}$ & $28.1 \pm 13.8 \mathrm{~kg}$ & $36.7 \pm 17 \mathrm{~kg}$ & $30 \pm 10.4 \mathrm{~kg}$ & $38.6 \pm 11.8 \mathrm{~kg}$ & $30.7 \pm 11.3 \mathrm{~kg}$ \\
\hline \multicolumn{2}{|c|}{ Cohen's D effect size } & \multicolumn{2}{|c|}{ Cohen's D effect size } & \multicolumn{2}{|c|}{ Cohen's D effect size } & \multicolumn{2}{|c|}{ Cohen's D effect size } \\
\hline \multicolumn{2}{|c|}{0.35} & \multicolumn{2}{|c|}{0.33} & \multicolumn{2}{|c|}{0.58} & \multicolumn{2}{|c|}{0.61} \\
\hline
\end{tabular}

\section{Discussion}

The present study attempted to evaluate the K-Force Grip dynamometer of K-Invent by comparing it with the gold standard Jamar. Regarding concurrent validity, both dynamometers exhibited excellent correlation coefficients as recommended by the literature. Specifically, Mathiowetz V. et al. [24] found similar results comparing the Rolyan dynamometer with the Jamar in a group of 60 healthy subjects.

The procedure of concurrent validity as shown by the absence of bias and also the fitting of the data produced results that were within the recommendations of the literature, which are considered acceptable by the American Society of Hand Therapists [25]. This finding supports the idea that the calibration process for both instruments is valid. In fact, this is of great importance, since the need for recalibration, even after a given time of use, would have been a great disadvantage for both instruments and a big discomfort for the users, most of whom may not be able to deliver the process. Furthermore, in both the $\mathrm{K}$-Force app and Jamar dynamometer, there is neither a calibration module nor directions for calibration in the manual. In the case of Jamar, the manual suggests that the device must be sent to the manufacturer for recalibration, elapsing one year of use, which is a procedure that can be very arduous for the user. However, it is suggested that, if a dynamometer is frequently used, calibration should take place at least every 5 months [26] following a detailed protocol measurement.

Intra-class correlations showed that intra-instrument reliability was excellent. Considering Jamar, this was a finding which is consistent with previous reports [27,28]. Regarding K-Force Grip, the high values of correlation within the trials proves the consistency of the instrument in measuring force. Along with the excellent concurrent validity found from the previous test, the use of K-Force Grip as a valid instrument for measuring hand grip strength is justified. Especially for the pain group, the present results for both instruments confirmed another interesting finding of the literature, that the reliability of grip strength is good among patients with shoulder impingement syndrome [29], enhancing the use of the test as a reliable quantitative method for the specific syndrome.

Intra-class correlations referring to inter-instrument reliability were good, indicating the similarity of K-Force Grip measurements to Jamar. This proves that K-Force Grip is a reliable device. It is also important that the above findings were observed for both groups. In fact, the pain group, despite the relative larger values of standard deviations, exhibited larger coefficients than the healthy group.

Despite the previously mentioned similarities, the measured values were significantly different between the two instruments for both groups, as shown by the paired $t$-tests. This observed difference could be attributed to the different width of the handle positions adopted between the two instruments. The second handle position in Jamar is supposed to be the most reliable and consistent position. It is the most appropriate position for producing maximal grip strength for the majority of the participants, as stated by previous studies that tested all five positions of Jamar [30,31]. 
In the present study, the second position was selected because of its similarity to K-Force Grip dimensions. Nevertheless, the effectiveness of the handle position should be, and actually is, related to the anthropometric parameters of the hand [32]. Therefore, since the dimensions of the handle were similar but not the same, force level differences are not an unexpected finding. Force level differences may occur apart from the handle position as a function of different testing postures, either by hand placement, or body position [33]. Lastly, an interesting descriptive observation mainly regarding the pain group was that they had similar average values for both hands, at least with the same instrument. This could be an indication that the participants' effort was not restricted by their shoulder impingement problem. That could be related to the position of their arm during the test, which was not a demanding one for their shoulder. Another point arising from this observation, concerning the functionality of the shoulder, is that, perhaps, grip strength should be tested under dynamic task conditions within the shoulder joint movement band [34]. This practice, in combination with kinematic information from the upper arm, should provide a more functional assessment of the problem and a more holistic evaluation of the rehabilitation process.

Overall, it is apparent that different handgrip positions and testing protocols may provide different findings that could be more useful for the clinical practice. Furthermore, the fact that K-Force Grip does not have an adjustable hand grip may influence, at some degree, the capacity of exerting grip strength for certain participants.

\section{Conclusions}

The critical role of the consistency of the measurement should be the consequential element in the measurement values, rather than their actual differences. Hence, since K-Force Grip was found to be a valid instrument for measuring the hand grip strength for both the healthy group and the pain group, the next important issue to be considered is the proper use of the instrument for the benefit of the users, especially for patients during a rehabilitation process. Nevertheless, a proportional bias between the instruments was apparent in the OLP test. As indicated by the extreme values of the outliers, it is important to consider these issues during data reduction and likely exclude them from the analysis. Yet, this should not be done automatically but under serious consideration whether or not these values may be a result of an adverse or uncomfortable experience with the instruments or a misunderstanding of the procedure from the participants. Nevertheless, in the present case, the outliers' exclusion would have helped to reveal a systematic bias between the instruments, as indicated in the last row of Table 2, and was likely expected considering the $t$-test. Therefore, practitioners need to account for systematic reduction in the obtained values when K-Force Grip is used.

As foretold, a training module is an important step for the use of hand grip dynamometers in the rehabilitation process. Along with the monitoring of performance through a database, it can be a useful tool for therapists and scientists. Such features are already utilized through K-Force Grip. Similar features are also available in the Jamar Smart, which is a wireless version of Jamar connected to a smartphone. As the next step, features allowing the user/therapist to easily build a complete custom rehabilitation protocol, according to the needs of his/her patient, would add value to the quality of hand grip skills rehabilitation, providing personalized protocols, which may have a beneficial and vital impact in a patient's life.

The present study apart from testing the reliability and validity of a handgrip dynamometer, attempted to reflect the evolution of the relevant technology and its implications on clinical and research settings. New approaches can be unrestricted by space and time limitations, or expert's presence during measurements and therapeutic sessions. Moreover, observation and study of evolving phenomena in real time through data series of grip strength and not just distinct values may offer a different perspective to the whole process of rehabilitation. 
Author Contributions: Conceptualization, methodology, validation, writing, review and editing supervision, formal analysis: T.N. Protocol development, investigation, writing, review and editing, measurements: S.S. and P.A. Methodology, data curation: C.C. Patient recruitment measurements, methodology, data curation: T.L. All authors have read and agreed to the published version of the manuscript.

Funding: This research received no external funding.

Institutional Review Board Statement: The study was conducted according to the guidelines of the Declaration of Helsinki, and approved by the Institutional Review Board (or Ethics Committee) of The Ethics Committee of the School of Physical Education and Sport Science at Thessaloniki (Aristotle University of Thessaloniki), approved this study. Approval number: EC-13/2020. Date of approval: 18 November 2020.

Informed Consent Statement: Informed consent was obtained from all subjects involved in the study. Written informed consent has been obtained from the patient(s) to publish this paper.

Data Availability Statement: The data presented in this study are available on request from the corresponding author.

Conflicts of Interest: The authors declare no conflict of interest.

\section{References}

1. Sousa-Santos, A.R.; Amaral, T.F. Differences in handgrip strength protocols to identify sarcopenia and frailty-A systematic review. BMC Geriatr. 2017, 17, 1-21. [CrossRef]

2. Bohannon, R.W. Muscle strength. Curr. Opin. Clin. Nutr. Metab. Care 2015, 18, 465-470. [CrossRef]

3. Horsley, I.; Herrington, L.; Hoyle, R.; Prescott, E.; Bellamy, N. Do changes in hand grip strength correlate with shoulder rotator cuff function? Shoulder Elb. 2016, 8, 124-129. [CrossRef] [PubMed]

4. Pizzato, T.M.; Baptista, C.R.J.A.; Souza, M.A.; Benedicto, M.M.B.; Martinez, E.Z.; Mattiello-Sverzut, A.C. Longitudinal assessment of grip strength using bulb dynamometer in Duchenne Muscular Dystrophy. Braz. J. Phys. Ther. 2014, 18, 245-251. [CrossRef] [PubMed]

5. Kachanathu, S.J.; Zedan, A.M.E.; Hafez, A.R.; Alodaibi, F.A.; Alenazi, A.M.; Nuhmani, S. Effect of shoulder stability exercises on hand grip strength in patients with shoulder impingement syndrome. Somatosens. Mot. Res. 2019, 36, 97-101. [CrossRef] [PubMed]

6. Ramlagan, S.; Peltzer, K.; Phaswana-Mafuya, N. Hand grip strength and associated factors in non-institutionalised men and women 50 years and older in South Africa. BMC Res. Notes 2014, 7, 8. [CrossRef]

7. Yorke, A.M.; Curtis, A.B.; Shoemaker, M.; Vangsnes, E. The impact of multimorbidity on grip strength in adults age 50 and older: Data from the health and retirement survey (HRS). Arch. Gerontol. Geriatr. 2017, 72, 164-168. [CrossRef]

8. Aguilar-Lazcano, C.A.; Rechy-Ramirez, E.J. Performance analysis of leap motion controller for finger rehabilitation using serious games in two lighting environments. Measurement 2020, 157, 107677. [CrossRef]

9. Friedman, N.; Chan, V.; Reinkensmeyer, A.N.; Beroukhim, A.; Zambrano, G.J.; Bachman, M.; Reinkensmeyer, D.J. Retraining and assessing hand movement after stroke using the MusicGlove: Comparison with conventional hand therapy and isometric grip training. J. Neuroeng. Rehabil. 2014, 11, 76. [CrossRef]

10. Confalonieri, M.; Guandalini, G.; Da Lio, M.; De Cecco, M. Force and touch make video games serious for dexterity rehabilita-tion. pHealth 2012, 177, 139-144. [CrossRef]

11. Guerra, R.S.; Amaral, T.F.; Sousa, A.S.; Fonseca, I.; Pichel, F.; Restivo, M.T. Comparison of Jamar and bodygrip dynamometers for handgrip strength measurement. J. Strength Cond. Res. 2017, 31, 1931-1940. [CrossRef]

12. Bellace, J.V.; Healy, D.; Besser, M.P.; Byron, T.; Hohman, L. Validity of the Dexter evaluation system's Jamar dynamometer attachment for assessment of hand grip strength in a normal population. J. Hand Ther. 2000, 13, 46-51. [CrossRef]

13. Amaral, J.F.; Mancini, M.; Júnior, J.M.N. Comparison of three hand dynamometers in relation to the accuracy and precision of the measurements. Braz. J. Phys. Ther. 2012, 16, 216-224. [CrossRef] [PubMed]

14. Hogrel, J.-Y. Grip strength measured by high precision dynamometry in healthy subjects from 5 to 80 years. BMC Musculoskelet. Disord. 2015, 16, 1-12. [CrossRef]

15. Eckman, M.; Gigliotti, C.; Sutermaster, S.; Mehta, K. Get a grip! Handgrip strength as a health screening tool. In Proceedings of the IEEE Global Humanitarian Technology Conference (GHTC 2014), San Jose, CA, USA, 10-13 October 2014; pp. $242-248$.

16. Agnew, P.; Maas, F. Jamar dynamometer and adapted sphygmomanometer for measuring grip strength in patients with rheumatoid arthritis. Occup. Ther. J. Res. 1991, 11, 259-270. [CrossRef]

17. Mathiowetz, V.; Vizenor, L.; Melander, D. Comparison of baseline instruments to the Jamar dynamometer and the B\&L engineering pinch gauge. Occup. Ther. J. Res. 2000, 20, 147-162. [CrossRef]

18. Shechtman, O.; Gestewitz, L.; Kimble, C. Reliability and validity of the DynEx dynamometer. J. Hand Ther. 2005, 18, 339-347. [CrossRef] 
19. Farfaras, S.; Ejerhed, L.E.; Hallström, E.K.; Hultenby, K.; Meknas, K.; Movin, T.; Papadogiannakis, N.; Kartus, J.-T. More histologic and ultrastructural degenerative signs in the subscapularis tendon and the joint capsule in male patients with shoulder impingement. Knee Surgery, Sports Traumatol. Arthrosc. 2017, 26, 79-87. [CrossRef] [PubMed]

20. Buckup, K. Clinical Tests for the Musculoskeletal System Examinations, Signs, Phenomena, 2nd ed.; Thieme: Stuttgart, Germany, 2008.

21. Su, C.Y.; Lin, J.H.; Chien, T.H.; Cheng, K.F.; Sung, Y.T. Grip strength in different positions of elbow and shoulder. Arch. Phys. Med. Rehabil. 1994, 75, 812-815. [CrossRef]

22. Savva, C.; Karagiannis, C.; Rushton, A. Test-retest reliability of grip strength measurement in full elbow extension to evaluate maximum grip strength. J. Hand Surg. Eur. Vol. 2012, 38, 183-186. [CrossRef] [PubMed]

23. Ludbrook, J. Special article comparing methods of measurement. Clin. Exp. Pharmacol. Physiol. 1997, 24, 193-203. [CrossRef] [PubMed]

24. Mathiowetz, V. Comparison of Rolyan and Jamar dynamometers for measuring grip strength. Occup. Ther. Int. 2002, 9, 201-209. [CrossRef]

25. Hamilton, A.; Balnave, R.; Adams, R. Grip strength testing reliability. J. Hand Ther. 1994, 7, 163-170. [CrossRef]

26. Fess, E.E. A method for checking Jamar dynamometer calibration. J. Hand Ther. 1987, 1, 28-32. [CrossRef]

27. Hamilton, G.F.; McDonald, C.; Chenier, T.C. Measurement of grip strength: Validity and reliability of the sphygmomanometer and Jamar grip dynamometer. J. Orthop. Sports Phys. Ther. 1992, 16, 215-219. [CrossRef] [PubMed]

28. Svens, B.; Lee, H. Intra- and inter-instrument reliability of Grip-Strength Measurements: GripTrack ${ }^{\mathrm{TM}}$ and Jamar ${ }^{\circledR}$ hand dynamometers. Br. J. Hand Ther. 2005, 10, 47-55. [CrossRef]

29. Savva, C.; Mougiaris, P.; Xadjimichael, C.; Karagiannis, C.; Efstathiou, M. Test-retest reliability of handgrip strength as an outcome measure in patients with symptoms of shoulder impingement syndrome. J. Manip. Physiol. Ther. 2018, 41, 252-257. [CrossRef]

30. Crosby, C.A.; Wehbé, M.A. Hand strength: Normative values. J. Hand Surg. 1994, 19, 665-670. [CrossRef]

31. Trampisch, U.S.; Franke, J.; Jedamzik, N.; Hinrichs, T.; Platen, P. Optimal Jamar dynamometer handle position to assess maximal isometric hand grip strength in epidemiological studies. J. Hand Surg. 2012, 37, 2368-2373. [CrossRef]

32. Kong, Y.-K.; Kim, D.-M. The relationship between hand anthropometrics, total grip strength and individual finger force for various handle shapes. Int. J. Occup. Saf. Ergon. 2015, 21, 187-192. [CrossRef]

33. El-Sais, W.M.; Mohammad, W.S. Biomechanical effect of testing positions on hand grip strength. J. Mech. Med. Biol. 2016, 16, 1650026. [CrossRef]

34. LaStayo, P.; Hartzel, J. Dynamic versus static grip strength: How grip strength changes when the wrist is moved, and why dynamic grip strength may be a more functional measurement. J. Hand Ther. 1999, 12, 212-218. [CrossRef] 